\title{
The Global Motions of the Cometary Knots in the Helix Planetary Nebula (NGC 7293)
}

\author{
J. Meaburn ${ }^{1}$, C.A. Clayton ${ }^{2}$, M. Bryce ${ }^{1}$ and J.R. Walsh ${ }^{3}$ \\ ${ }^{1}$ Department of Physics \& Astronomy, University of Manchester; \\ ${ }^{2}$ Rutherford Appleton Laboratory; ${ }^{3}$ ESTCF, ESO, Garching bei München
}

The nature and origin of the hundreds of ionized knots, with cometary tails, in the Helix planetary nebula are both being unravelled by recent observations. Healey and Huggins (1990, AJ, 100, 511), Meaburn et al (1992, MNRAS, 255, 177) and Walsh and Meaburn (1993, ESO Messenger, 73, 35) all showed, in various ways, that these knots have dense $\left(10^{6} \mathrm{~cm}^{-3}\right)$, dusty, molecular cores with ionized arcs of gas on the surfaces pointing towards the ionizing star. This structure is confirmed in detail by the HST imagery of O'Dell and Handron (1996, ApJ, in press).

Systematic measurements of the radial velocities of a significant sample of the Helix cometary knots have been made in a sustained period of exceptionally good 'seeing'. This kinematical information has been combined with [O III] 5007 $\AA$ NTT (ESO) images of the whole of the Helix nebula to identify the location of each knot. Meaburn et al (1992) had shown that nearside knots appeared as dark patches on [O III] $5007 \AA$ images as their dusty cores scattered the central [O III] 5007 $\AA$ emission of the Helix nebula. A global expansion velocity, assuming radial expansion in a spherical shell, of approx $10 \mathrm{~km} \mathrm{~s}^{-1}$ for the system of knots has been found which is far less than the $24 \mathrm{~km} \mathrm{~s}^{-1}$ general expansion of the Helix nebula. A model is favoured where the cometary knots are relics of condensations in the precursor, Red-Giant/AGB, slow, dense, wind now overrun by faster shells (and perhaps fast winds) generated after the formation of the planetary nebula. More complex models for the global structure of the system of knots are also being considered. 